\title{
Tracing international migration in projections of income and inequality across the Shared Socioeconomic Pathways
}

\author{
Hélène Benveniste ${ }^{1,2}$ (D) Jesús Crespo Cuaresma ${ }^{1,3,4,5}$ • Matthew Gidden ${ }^{1,6}$. \\ Raya Muttarak ${ }^{1,4}$
}

Received: 30 September 2020 / Accepted: 24 May 2021/Published online: 9 June 2021

(C) The Author(s) 2021

\begin{abstract}
The Shared Socioeconomic Pathways (SSPs) represent five narratives of future development used for climate change research. They include quantified projections of socioeconomic variables such as population, income levels, inequalities, and emissions over the twenty-first century. The SSP's population projections embody explicit, pathway-specific international migration assumptions, which are only implicit in the projections of other variables. In this contribution, we explicitly quantify the effects of international migration on income levels and income inequality across and within countries by comparing the original SSP projections to scenarios of zero migration. Income projections without migration are obtained by removing two effects of migration on income dynamics: changes in population size and remittances sent to origin countries. We base our remittance estimates on migrant stocks derived from bilateral migration flow estimates obtained from a gravity model. We find that, on average, migration tends to make the world richer in all SSP narratives. The nature of migration and remittance corridors is shaped by the specific scenario of future development considered. Depending on the particular SSP narrative and world region considered, the effects of migration on income can be substantial, ranging from -5 to $+21 \%$ at the continental level. We show that migration tends to decrease income inequality across countries and within country in most destination countries but does not affect within-country inequality in origin countries. This new set of projections is consistent with the interdisciplinary framework of the SSPs, which makes it particularly useful for assessing global climate and sustainable development policy options.
\end{abstract}

Keywords International migration · Shared Socioeconomic Pathways $\cdot$ Income $\cdot$ Inequality

Hélène Benveniste

helene.benveniste@princeton.edu

Extended author information available on the last page of the article 


\section{Introduction}

Migration decisions are often multi-causal and environmental stress is merely one of many underlying drivers of migration. Environmental change is likely to influence migration through various economic, political, social, demographic, and environmental channels (Black et al. 2011a, b). Both extreme weather events, whose intensity is expected to increase in the future, and slow-onset events (e.g., droughts, sea-level rise (Desmet et al. 2021) influence migration patterns. These environmental changes might enhance migration when used as an adaptation strategy or suppress migration when subgroups of vulnerable population cannot afford outmigration in the future (Borderon et al. 2019, Cattaneo and Peri 2016; Hoffmann et al. 2020).

In the context of analyzing the effect of environmental change on societal outcomes, migration has commonly been interpreted as a potential coping strategy to external shocks, including weather shocks (Black et al. 2011a, b, Castells-Quintana et al. 2018). It is therefore possible that migration is increasingly used as an adaptation strategy to climate change (Gemenne and Blocher 2017, Kanta et al. 2018). Migration may also affect aggregate labor productivity and lead to welfare gains for migrant households through relocation of labor from less productive areas (e.g., rural hinterlands) to more productive locations (e.g., urban areas). Likewise, remittances from earlier migrants as a diversified source of income can be used as a means to mitigate and cope with climate shocks (Arezki and Brückner 2012) and may reduce incentives for aspiring migrants to move. Changes in migration patterns, including those driven by climate change, thus have substantial implications for future socioeconomic development.

Migration dynamics interact strongly with other socioeconomic development dimensions. Migration is not only a key component in global population dynamics (Cohen et al. 2008, Lutz and KC 2010, Azose and Raftery 2015), but it also affects and is affected by economic development. On the one hand, migration is shaped by economic development, which acts both as a pull and push factor of labor mobility. On the other hand, international migrants bring about socioeconomic changes in both origin and destination countries through remittances, labor force participation, and its effect on economic growth (de Haas 2010, Hanson and McIntosh 2016, Clemens and Pritchett 2008). Thus, changes in migration patterns can strongly influence income levels and their distribution across and within countries. Likewise, migration also contributes to shifts in carbon emission transfers (Liang et al. 2020; Qi and Li 2020) through its contribution to population growth and changing energy consumption patterns. Therefore, there is a need for projections of future socioeconomic development trajectories that explicitly incorporate changes in migration patterns.

Given the potential effects that migration can have on well-being at the global level, a clear understanding of interactions between international migration patterns and future environmental change appears particularly relevant for assessing current global policy options to combat the negative effects of climate change. In order to anticipate societal challenges posed by climate change, it is important to consider how the global climate system will evolve, as well as the degree of vulnerability and capacity of societies to mitigate and adapt to future environmental change. However, while climate modelling has witnessed major progress in predicting global warming trends and their consequences on the biosphere, until recently only humble efforts had been undertaken to design plausible future scenarios of socioeconomic and human development (Lutz and Striessnig 2015). This imbalance partly owes to the high level of uncertainty in the future development of various socioeconomic factors, rendering the quantification of such scenarios particularly difficult. 
With the aim of exploring uncertainties in future environmental and societal change in a more systematic manner, a number of qualitatively distinct scenarios have been developed in the framework of the Intergovernmental Panel on Climate Change's (IPCC) fifth assessment report. The Shared Socioeconomic Pathways (SSPs) represent five qualitative narratives of future global development, and have proven to be useful tools to study heterogeneous futures in the context of climate change research. They highlight five different combinations of challenges to climate change mitigation and adaptation. Embedded in these narratives are assumptions on future international migration. Some key socioeconomic variables have been quantitatively projected within the SSP framework up to the year 2100. These include population by age and educational attainment at the country level (KC and Lutz 2017) and by urban/rural characteristics at the $1 / 8^{\circ}$ level (Jones and O’Neill 2016), gross domestic product (GDP, Dellink et al. 2017, Crespo Cuaresma 2017, Leimbach et al. 2017), income inequality (Rao et al. 2019), final energy consumption, and greenhouse gas emissions (Riahi et al. 2017, van Vuuren et al. 2017, Fricko et al. 2017, Fujimori et al. 2017, Calvin et al. 2017, Kriegler et al. 2017). The population component reflects explicit pathway-specific migration assumptions and is used as an input to all other components. On the other hand, migration assumptions are only implicit in the other components for which quantitative projections exist. To our knowledge, existing SSP quantification exercises are done separately for each socioeconomic component and do not account for the possible interactions between the variables.

In this contribution, we exploit the framework provided by the SSP projections to quantify the effects of international migration on future trajectories of GDP and between-countries income inequality, as well as on the distribution of income within countries. We assess the role of migration in future scenarios of development by comparing original projections to scenarios where zero migration is assumed. We show that migration tends to make the world richer, on average, in all SSP scenarios. Furthermore, the nature of migration and remittance corridors is significantly influenced by the scenario of future development considered. Depending on the narrative and location, the migration effect on income can be substantial, ranging from -5 to $+21 \%$ at the continental level. Moreover, we show that migration tends to make the world more equal in terms of income for all SSP scenarios at all times, increases inequality in few origin countries, and reduces inequality in most destination countries. Here as well, the scenario considered significantly affects the magnitude of the migration effect on income inequality.

With this contribution, we do not aim to propose plausible predictions of future migration under climate change, but rather to highlight where and to what extent migration plays a role in projections of income and inequality that are widely used in climate change research. The conceptual nature of the SSP framework implies that the projections are not meant to be accurate predictions, but are instead internally consistent scenarios that do not integrate the effects of climate change (see Riahi et al., 2017). The new set of projections developed here, ensuring comparability with the original set of existing projections, is perfectly coherent with the SSP's interdisciplinary methodological framework. Our quantification of migration patterns by SSP scenario presents a particularly useful addition to the pool of instruments aimed at the assessment of global climate and migration policy options. In particular, our projections without migration can be used as a consistent input of models combining explicit representations of migration, economic activity, and climate change (e.g., Benveniste et al. 2020).

The paper is organized as follows. Section 2 summarizes the SSP framework and presents the different scenarios used to describe future global developments. Section 3 describes the methods we employ to quantify the role of migration flows in projections of population and GDP. Section 4 presents the data employed. Section 5 discusses our resulting projections of the explicit effect of migration. Finally, Section 6 concludes. 


\section{Migration, GDP, and inequality in the Shared Socioeconomic Pathways}

The Shared Socioeconomic Pathways (SSPs) offer five qualitative narratives of future global development. They highlight different combinations of challenges to mitigation and adaptation to climate change, structured into five scenarios. The first scenario, SSP1 ("Sustainability"), describes a sustainable future, focused on strengthening well-being, where the world features low inequalities and strong international cooperation. In this world, migration is assumed to stay at medium levels, i.e., in line with historical patterns. The SSP2 ("Middle of the Road") scenario features a world where historical patterns and trends are assumed to persist in the future in a context of political stability but with limited social cohesion. In this scenario, migration is also assumed to stay at medium levels. SSP3 ("Regional Rivalry") showcases a fragmented world with regional conflicts occurring and a strong emphasis on security, in particular on closed borders; in such a scenario for future developments, a low level of migration is assumed. SSP4 ("Inequality") assumes a highly unequal world, with strong inequalities both across and within countries, and the emergence of a global elite. For this scenario, migration is assumed to stay at medium levels. Note that the characteristics of migrants (in terms of, for example, income levels or skills) in this scenario are quite different from those of migrants in more optimistic scenarios such as SSP1 and SSP2. SSP4 assumes a polarized education distribution with a subgroup of very highly educated population concentrating in member countries of the Organisation for Economic Co-operation and Development (OECD), while the majority in the rest of the world have low education. Finally, SSP5 ("Fossil-fueled Development") represents a future of strong resource-intensive technological progress and economic growth, featuring a sustained use of competitive markets and widespread globalization. In this world, migration is assumed to be high (Abel 2018b, KC and Lutz 2017). Appendix Fig. A2 (based on O'Neill et al. 2017) presents a graphical scheme summarizing the combinations of challenges to adaptation and mitigation implied by each SSP narrative, together with the assumptions concerning global migration for each scenario.

These narratives have been translated into quantified paths of population, GDP, and inequality measures over the twenty-first century, but a differentiated assessment of the contribution of migration has not yet been carried out hitherto in the existing literature. Such an effort requires quantitative assessment of the effects of migration on population dynamics across countries, and explicitly modelling how economic developments are expected to change global migration corridors. Additionally, changes in remittances associated with such migration patterns and their projected effect on GDP per capita need to be incorporated into income projections, and may also affect within-country income inequality. In the following section, we present the methods employed to quantify the role of migration in each one of these variables.

\section{Methods}

\subsection{Migration in SSP population projections}

$\mathrm{KC}$ and Lutz (2017) provide population projections for the five narratives that constitute the scenarios within the SSP framework. The five SSP narratives presented above provide qualitative narratives on future global developments, which are translated to quantifications of population trajectories at the country level by means of assumptions on the joint dynamics of educational attainment, fertility, mortality, and migration. The SSP1 scenario rests on the 
assumption that investments in education and health lead to an acceleration of the demographic transition worldwide. This translates to a low mortality and high education scenario, where fertility is assumed to decrease in the developing world but not to decline further in developed economies. The narrative associated to the SSP2 scenario corresponds to a middle-of-the-road benchmark population trajectory, with medium fertility and mortality assumptions coupled with a continuation of the trends in educational attainment observed historically. SSP3 is a scenario of high population growth in the developing world and small changes in population in industrialized economies. High mortality and low education improvement is assumed worldwide, while fertility is assumed to remain low in the OECD countries and high in the rest of the world. The SSP4 scenario is characterized by polarization, with persistent high fertility and high mortality in high-fertility countries and low fertility and medium mortality in the rest of the world. Finally, SSP5 builds upon high education and low mortality assumptions for all countries, together with high fertility for industrialized countries and low fertility for all other economies.

Migration assumptions are also included in the SSP scenarios. In the original population projections by KC and Lutz (2017), ${ }^{1}$ the medium migration scenario (used in SSP1, SSP2, and SSP4) assumes constant in-migration and out-migration rates for the coming century. The high migration scenario (SSP5) essentially assumes that migration rates double by 2030 and then remain constant, while the low migration scenario (SSP3) assumes that migration rates converge to zero by 2030. In all scenarios, the number of people moving to another country in any given 5 -year period of the projection stays under $1 \%$ of global population. The overall immigrant share in global population is depicted in Appendix Fig. A3 for the selected 5-year periods.

Population projections under a zero migration assumption have also been developed in the context of the update of SSP projections (Lutz et al. 2018). ${ }^{2}$ Some features of these new counterfactual projections are discussed in Appendix Section A d) and illustrated in Appendix Fig. A4. We use these population projections to develop new projections of GDP with zero migration, explicitly quantifying the effect of migration dynamics on income differences in the SSP projections.

\subsection{Migration, remittances, and GDP in the SSP projections}

Our adjustment of GDP is carried out making use of the GDP projections along the SSP narratives developed by Dellink et al. (2017) as a starting point. Dellink et al. (2017) use an aggregate production function specification where GDP is linked to population dynamics, changes in total factor productivity, and physical and human capital accumulation, as well as energy and fossil fuel resources. The effect of migration is included implicitly in these GDP scenarios, as the original population projections are used as an input to construct them. We construct counterfactual income projections under the zero migration assumption, correcting the original projected income data by accounting for two effects of migration on GDP dynamics. First, the change in country-level population affects GDP through the changed population size. Second, remittances sent by migrants to their origin countries are also factored

\footnotetext{
${ }^{1}$ We use version 2 of the SSP population projections, by the same authors. These latest projections are forthcoming; a working paper is available in KC (2020). A description of the projection exercise can be found in Lutz et al. (2018).

2 These are not yet publicly available, but can be obtained upon request from Samir KC.
} 
into GDP levels in the projection period. Incorporating these effects in GDP per capita projections implies that the income variable including the effects of migration is given by ${ }^{3}$

$$
\begin{gathered}
G D P_{i, \text { mig }}(t)=G D P_{i, \text { nomig }}(t)\left(1+\frac{\operatorname{pop}_{i, \text { mig }}(t)-\text { pop }_{i, \text { nomig }}(t)}{\operatorname{pop}_{i, \text { nomig }}(t)}\right)+\operatorname{rem}_{i}(t), \\
y p c_{i, \text { mig }}(t)=y \mathrm{p} c_{i, \text { nomig }}(t)+\frac{\operatorname{rem}_{i}(t)}{\operatorname{pop}_{i, \text { mig }}(t)},
\end{gathered}
$$

where $\operatorname{pop}_{i}(t)$ represents the population size of a given country at time $t, G D P_{i}(t)$ is the total income, $y p c_{i}(t)$ denotes per capita income, and $\operatorname{rem}_{i}(t)$ is the net remittance flow into the country, while the subindices mig and nomig denote the scenarios with migration flows and assuming zero migration, respectively.

Adjusting GDP figures for net remittance flows requires the estimation of bilateral migration and remittance flows over the projection period. The original SSP population projections do not provide explicit bilateral migration flows, although in- and out-migrant flows are implicitly included in them. In order to obtain projections of flows of remittances, we use an estimated gravity model (Jones and O'Neill 2013, Jones and O'Neill 2016) that summarizes the quantitative effects of push and pull factors on migration flows. We consider two different specifications of our model, one for absolute migrant flows and another one for the share of migrants from a given country in the origin population size. In this framework, the number of people moving from one country to another is assumed to depend on population size $(p o p(t))$ for the specification in levels and the ratio of population densities (popdens $(t))$ for the specification in ratios, economic opportunities, here measured as per capita income $(y p c(t))$, and the corresponding ratio in the alternative specifications, as well as geographic proximity between the two countries, here featured as the geodesic distance between capital cities $\left(\right.$ dist $\left._{i j}\right)$. We also include a set of bilateral characteristics of the origin/destination pair summarized in the vector $\Lambda_{i j}$, which includes (a) an indicator variable measuring whether the two countries share a common official language and (b) the share of migrant's income sent as remittance and the cost of sending the remittances, which proxy existing migrant networks as well as remittance costs and benefits. We estimate bilateral migrant flows between countries $i$ and $j\left(m o v e_{i j}\right)$ as well as their ratios to origin population ( move $_{i j} /$ pop $_{i}$ ) by making use of gravity models specified as follows,

$$
\begin{aligned}
\operatorname{logmove}_{i j}(t)= & \beta_{0}+\beta_{1} \log \operatorname{pop}_{i}(t)+\beta_{2} \log p o p_{j}(t)+\beta_{3} \log y p c_{i}(t)+\beta_{4} \log y p c_{j}(t) \\
& +\beta_{5} \log \text { dist }_{i j}+\Gamma \Lambda_{i j}+\epsilon_{i j}(t) \\
\log \left(\frac{\text { move }_{i j}(t)}{\operatorname{pop}_{i}(t)}\right)= & \theta_{0}+\theta_{1} \log \left(\frac{\text { popdens }_{j}(t)}{\text { popdens }_{i}(t)}\right)+\theta_{2} \log \left(\frac{y p c_{j}(t)}{y p c_{i}(t)}\right)+\theta_{3} \log \text { dist }_{i j}+\Psi \Lambda_{i j} \\
& +\nu_{i j}(t)
\end{aligned}
$$

\footnotetext{
${ }^{3}$ Note that we abstract from modelling effects on GDP growth caused by differential productivity of migrants. In addition, we work with the assumption that migration dynamics do not affect country-specific total factor productivity dynamics (Ortega and Peri 2009). Equations (1) and (2) are thus identities that do not take into account the potential effect of migration beyond the adjustment via remittances. Such an exercise goes beyond the scope of our analysis here, but would be a fruitful avenue for future research.
} 
where $\varepsilon_{i j}$ and $v_{i j}$ are error terms which fulfill the standard assumptions of the normal linear regression model.

The parameters in Eq. (3) and Eq. (4) are estimated using data for the period 1990-2015 (see Section 4.1) and are used to obtain migration projections. The total number of migrants leaving a given country at time $t$ is given by leave $e_{i}(t)=\sum_{j}$ move $_{i j}(t)$, while the number of immigrants is given by enter $(t)=\sum_{i}$ move $_{i j}(t)$.

We ensure consistency with the original SSP population projections by rescaling the bilateral flows derived from our gravity model, so that for each country the number of immigrants over a given 5-year period equals that implied by the original population projections in each SSP narrative. We further compute a state variable $\left(\operatorname{stock}_{i j}(t)\right)$ that keeps count of how many migrants from one region are present in another region at a given time. We assume that only first-generation migrants send a share of their income back to their origin country in the form of remittances for the duration of their life. This assumption coarsely illustrates empirical findings of the migration literature focusing on second-generation remittances, suggesting that second-generation migrants are significantly less likely to send remittances back to their country of origin and send smaller amounts (Fokkema et al. 2013). This duration is computed as life expectancy at birth $\left(\lambda_{i}(t)\right)$ in the destination region (Wallace et al. 2019; Loi and Hale 2019) minus median age of migrants at the time of migration $\left(\mu_{i}(t)\right)$,

$$
\operatorname{stock}_{i j}(t)=\operatorname{stock}_{i j}(t-1)+\text { move }_{i j}(t)-1_{t>\lambda_{j}(t)-\mu_{j}(t)}\left[\operatorname{enter}_{j}\left(t-\lambda_{j}(t)+\mu_{j}(t)\right)\right]
$$

We derive remittance flows from bilateral migrant stocks by assuming that migrants send a share $\tau_{i j}(t)$ of their income to their origin region, for a cost $\psi_{i j}(t)$. We use this state variable to calculate remittances received in a given country $\left(\operatorname{rem}_{i}(t)\right)$ at period $t$ as

$$
\operatorname{rem}_{i}(t)=\sum_{j} \operatorname{stock}_{i j}(t) y p c_{j}(t) \tau_{i j}(t)\left(1-\psi_{i j}(t)\right)-\sum_{k} \operatorname{stock}_{k i}(t) y p c_{i}(t) \tau_{\mathrm{ki}}(t)\left(1-\psi_{k i}(t)\right)
$$

The calibration of the parameters in Eqs. (5) and (6) is described in detail in Section 4.2.

\section{Data}

\subsection{Calibration of the migration gravity equation}

We estimate the parameters in our gravity models (Eqs. (3) and (4)) using data on migration flows between countries for 1990-2015 from Azose and Raftery (2019) as compiled in Abel and Cohen (2019). In order to ensure robustness, we also estimate the parameters of the gravity model using another dataset for the same period sourced from Abel (2018a). Both datasets on migration flows are derived from data on migrant stocks from the World Bank using demographic accounting methods. We use population data for the same period from the Wittgenstein Centre (Lutz et al. 2019) and GDP per capita data from the World Bank's World Development Indicators. We compute distances between countries as distances between their respective capital cities. Our models are estimated based on 76,358 or 94,459 observations, depending on the dataset used for bilateral migration flows. For the projection exercise, we use the dataset from the Wittgenstein Center for population and the IIASA SSP database for GDP, both of which are available for 2010-2100 in 5-year intervals.

Our parameter estimates for the bilateral migration models employed are presented in Table 1 . The estimates obtained by exploiting variation across countries and over time (that 
Table 1 Results from OLS regression on migration flows (columns 1, 2, and 5 following Eq. 3) and migrants to origin population ratios (columns 3 and 4 following Eq. 4). Specifications with year fixed effects (all columns), and origin and destination fixed effects (columns 2 and 4). Estimates in columns 1-4 are obtained using data from Azose and Raftery (2019) and Abel and Cohen (2019), while estimates in columns 5 are obtained based on data from Abel (2018a). Standard errors clustered at the origin and destination levels are in parenthesis

Azose and Raftery (2019)

Migration flows

(1)

$\begin{array}{ll}0.686 * * * & 0.746 * * \\ (0.040) & (0.235) \\ 0.681 * * * & -0.748 * * \\ (0.042) & (0.235)\end{array}$

Population densities ratio

Origin per capita GDP

Destination per capita GDP

$0.428 * * *$

(0.059)

$0.837 * * *$

(0.069)

Ratio of per capita GDP

Distance between countries

Remittances as share of income

Cost of sending remittances

Common official language

Year FE

Origin FE

Destination FE

Estimator

N

R2

Within-R2
(2)

0.155

(0.134)

$-0.004$

(0.097)

$\begin{array}{ll}-1.291 * * * & -1.472 * * * \\ (0.061) & (0.062) \\ 0.155^{*} & 0.190^{*} \\ (0.070) & (0.077) \\ -9.418 & -12.894 \\ (15.597) & (15.027) \\ 1.727 * * * & 1.606 * * * \\ (0.130) & (0.128) \\ \text { Yes } & \text { Yes } \\ & \text { Yes } \\ & \text { Yes } \\ \text { OLS } & \text { OLS } \\ 76,358 & 76,358 \\ 0.478 & 0.610 \\ 0.477 & 0.370\end{array}$

Migrants ratio

(3)

(4)

Abel (2018a)

Migration flows

(5)

$0.575 * * *$

(0.031)

$0.602 * * *$

(0.042)

$\begin{array}{ll}0.037 & -0.249 \\ (0.071) & (0.148)\end{array}$

$0.105 *$

(0.044)

$0.791 * * *$

(0.065)

$\begin{array}{ll}0.145^{*} & -0.088 \\ (0.072) & (0.084) \\ -1.166^{* * *} & -1.472 * * * 4\end{array}$

$-1.166 * * * \quad-1.472 * *$

$(0.084) \quad(0.062)$

$-0.053$

$0.190 *$

$-1.038 * * *$

(0.061)

(0.088)

$-10.532$

(19.234)

$1.217 * * *$

(0.077)

$-12.891$

(15.030)

$1.607 * * *$

(0.128)

(0.214)

Yes

Yes

Yes

Yes

OLS OLS

76,358

0.176

76,358

0.616

0.369
0.090

(0.085)

$-15.333$

(15.765)

$1.439 * * *$

(0.119)

Yes

OLS

94,459

0.410

0.402

$* p<0.05, * * p<0.01, * * * p<0.001$

is, without origin and destination fixed effects, see column 1) imply that migration flows between two countries increase with the origin population size and with the existence of a common language, and decrease with the distance between countries, as suggested in other studies using similar models (e.g., Cohen et al., 2008). ${ }^{4}$ Migration flows also tend to increase with destination population size and per capita GDP. When including origin and destination fixed effects (column 2), the inference is based on deviations of migration flows from originand destination-specific averages and the direction of the effect of destination population size and per capita GDP change and lose significance. For our alternative model with the migrant ratio as a dependent variable, the coefficient for population density indicates that denser areas tend to act as a pull factor, potentially due to higher productivity (coefficient without country

\footnotetext{
${ }^{4}$ The importance of language proximity as a determinant of migration flows has been studied in detail by Adsera and Pytlikova (2015), and the inclusion of covariates that account for such cultural similarities is commonplace in gravity specifications for migration flows. Assessing the determinants of migration, Poprawe (2015) finds common language to be a robust covariate using extreme bounds analysis methods.
} 
fixed effects positive in column 3), but are eventually subject to congestion effects (coefficient with country fixed effects negative in column 4). Similarly, the coefficient attached to the ratio of GDP per capita indicates that individuals tend to move to countries with higher incomes (positive coefficient in column 3), but might require a minimum level of resources to be able to move (positive coefficient in column 2 and negative in column 4). The effects of bilateral characteristics (distance, remittance shares and costs, common official language) are similar in sign, size, and level of significance in all specifications. Unsurprisingly, remittance costs reduce migration, while the variable measuring remittances as a share of income has a positive effect on migration, hinting at the effects of migration networks on the persistence of migration flows.

The interpretation of the model with country fixed effects differs from that of the model without them, which explains the difference in the effects found across the two specifications. The inclusion of origin and destination fixed effects implies that we are considering effects that are inferred from deviations of migration flows from origin- and destination-specific long-run equilibria. The effects obtained in these models can thus be thought of as summarizing short- to medium-term elasticities. On the other hand, effects on changes in the long-term equilibria are factored into the parameter estimates of the model without the country fixed effects. We consider our model including only year fixed effects to be the most appropriate for this projection exercise. In addition, projecting timeinvariant country fixed effects would lead to flow differences across countries remaining constant in the long run, eventually hiding potential long-term effects of our socioeconomic covariates. We test several combinations of fixed effects for robustness and show results including origin and destination fixed effects in Appendix Section D. While some migration and remittance flows differ, the overall conclusions are not qualitatively different. We provide a further robustness check by estimating parameters using data from Abel (2018a) (see column 5) and find that our projections are virtually not affected by the data source. We provide a final robustness check by testing for reverse causality of migration on income at origin, in particular through remittances, by using income minus remittances received as an independent variable (see Appendix Table S1); we find that the coefficients are not affected.

\subsection{Calibration of remittance parameters}

The estimation of remittance flows is based on the assumption that only first-generation migrants send remittances (assumed to be a constant share of their income), for the rest of their lives after migration (Fokkema et al. 2013). Remaining lifetime duration is given by life expectancy in the destination country minus the age of migrants at the time of migration. While migrants do not necessarily have the same life expectancy as native-born populations, the existing empirical literature shows that significant heterogeneity across ethnic groups and origin countries exists (Singh and Miller 2004; Mehta et al. 2016). Mehta et al. (2016), for instance, find that some groups (e.g., Asian-born) have higher life expectancy than the native born in the USA, whereas migrants born in Central America, Europe, and Africa display similar life expectancy. Although a systematic review and meta-analysis of empirical studies by Aldridge et al. (2018) finds an overall mortality advantage among international migrants compared with native populations, this evidence is limited only to high-income destination countries and excludes marginalized subgroups of migrants. Given the reported heterogeneities of migrants' health status, we assume similar life expectancies of foreign-born populations to those of the native-born, since migrants' health outcomes and mortality patterns do tend to converge to those of the natives' as duration of stay increases (Wallace et al. 2019; Loi and Hale 2019). 
For the estimation, we use the SSP projections of life expectancy available from the Wittgenstein Centre for the period 1970-2100. Life expectancy values vary across countries from 34.2 to 74.9 years in 1970, and span 58.7 to 92.2 years in the most pessimistic scenario (SSP3) and 79.6 to 110 years in the most optimistic scenarios (SSP1 and SSP5) in 2100. To calibrate the age of migrants at the time of migration, we proceed as follows. First, we use immigration flows from the SSP population projections, available per 5-year age group. The resulting age distribution of migrants at the time of migration is very similar across countries, periods, and SSP narratives, thus ensuring consistency ( $\mathrm{KC}$ and Lutz 2017). In particular, approximately $17 \%$ of migrants are under 15 years old when migrating, $50 \%$ is between 15 and 30 years old, $24 \%$ is between 30 and 45 years old, and $9 \%$ above 45 . Data on the initial (current) stock of migrants by age group are derived using bilateral migrant stocks from the World Bank for the year 2017. We apply an average of two age distributions in each destination country to the current stocks data: the age distribution of migrants at the time of migration in the period 2015-2020, and the age distribution of overall destination population in the same period. Both are sourced from the SSP population projections (KC and Lutz 2017).

In Eq. (6), the share of income sent as remittances and costs are measured using data from the World Bank for 2017. We maintain these constants over time and across the SSP narratives, but we make them specific to each origin/destination pair. In order to calibrate the share of income sent as remittances, we use bilateral remittance estimates, bilateral estimates of migrant stocks, and per capita GDP data for 2017, the last year for which information for all three variables are available. For each origin/destination pair with missing data, we assign zero remittances. We assume the migrant's income to be $\max (y p c i(t)+y p c j(t) /$ 2, ypci(t)) and use it to compute $\tau(t)$. The range [0.0,0.28] covers the central $90 \%$ of the estimates obtained, which implies that migrants in $90 \%$ of origin/destination pairs send remittances amounting to between 0 and $28 \%$ of their yearly income. The cost of sending remittances is calibrated using the total cost of transactions in percentage of GDP from the Remittance Prices Worldwide database for 2017. For each origin/destination pair with missing data, we use the mean cost of all available pairs. We obtain an overall $90 \%$ interval for remittance costs of $[0.02,0.12]$.

\section{Results}

\subsection{Bilateral migration and remittance flows}

We use the estimates from the gravity model given by Eq. (3) to generate projections of bilateral migration flows between countries, which we rescale to SSP totals for the period 2015-2100 and for each one of the five SSP scenarios. We present these flows in the form of a heat map aggregated at the continental level for 2095 in Appendix Fig. B1.

Independently of the scenario chosen, we find that migrants tend to move within continents, as highlighted by the dark colors along the diagonal. Migration corridors from Africa to Asia and Europe and from Latin America and the Caribbean to Northern America are also present in the projected migration patterns. Migrant numbers increase from SSP1 to SSP2 and to SSP5 in a similar fashion across migration corridors. For SSP4, bilateral migration patterns are slightly different. As compared to other SSP scenarios, less intensive migration activity is observed along certain corridors (e.g., within Africa), while others present more migrants (e.g., from Asia to Europe). As expected, there is little effect of migration on population dynamics within 
the SSP3 scenario. When relating absolute migrant numbers to population sizes in origin regions, we find that migration to Northern America from all continents apart from Asia is the most significant one (while staying under $0.3 \%$ of the origin region population). The persistence of a high share of migrants within Europe is not surprising given the historical patterns, characterized by free mobility policy within a large part of the continent. When relating absolute migrant numbers to the population size of destination regions, migration within Northern America stands out, while migration from Asia to Oceania reaches significant levels. Similarly, migrants as share of destination population stay under $0.3 \%$ worldwide.

We present the projected remittance flows aggregated at the continental level for 2095 in Appendix Fig. B2. We find that most remittances are sent within continents, and remittance corridors between Europe, Asia, and Africa, and from Northern America to Latin America and the Caribbean can be recognized in the data, in line with the results for migration flows. Remittances increase from SSP2 to SSP1 and to SSP5. The inversion between SSP1 and SSP2 in terms of the relative amounts of migrants and remittances in the Asia-Africa corridor is due to both regions being richer yet less populated in SSP1 than in SSP2. For SSP4, bilateral remittance patterns are somewhat different. Some corridors see less transfers relatively to other SSP scenarios (e.g., within Africa), while some others present more transfers (e.g., from Latin America to Asia). As expected, there is a relatively small flow of remittances in SSP3. When relating absolute remittance amounts to GDP in receiving regions, we find that remittances sent in the same corridors are the largest ones (although under 10\% of GDP in the origin region). When relating absolute remittance amount to sending region GDP, remittances sent from Oceania, in particular to Asia, reach significant levels. Similarly, remittances as a share of GDP in the destination location tend to stay under $10 \%$.

\subsection{GDP per capita projections for zero migration: global outlook}

Based on our estimates obtained from Eq. (1), we produce country-specific projections of GDP and GDP per capita for the period 2015-2100 for each of the five SSP narratives under the assumption of zero migration. Our results at the global scale are illustrated in Fig. 1 (see Section B in the Appendix for country-level projections). Figure 1 a shows average global per capita GDP with (circles) and without (triangles) remittances, for the different SSP narratives. While the effect of migration dynamics is visible in the data, it is moderate and does not modify the ranking of income levels among the SSP narratives: SSP5 ("Fossil-fueled Development") is still the scenario with the consistently highest per capita GDP, and SSP3 ("Regional Rivalry") is the one with the lowest.

Figure $1 \mathrm{~b}$ shows relative changes in average per capita GDP with migration compared to our zero-migration projections, at the global level. We find that for all SSP scenarios, the average global per capita GDP is consistently higher with migration than without. The effect becomes substantial in the second half of the century for SSP4 ("Inequality"), where income levels increase above $5 \%$ by 2065 . Unsurprisingly, given the fact that the original SSP3 projections already include low migration levels, the effects are particularly small for this scenario. For SSP1, SSP2, and SSP5, migration does not lead to further sizeable increases in GDP per capita at the global level, stabilizing at a difference of $2-3 \%$ by the end of the century. This stark contrast in the evolution of the migration effect on income levels is influenced by the underlying assumptions concerning worldwide convergence of income levels across the narratives. In an SSP4 world, dominated by global inequalities, strong differences in per capita income between countries tend to persist all along the century. 


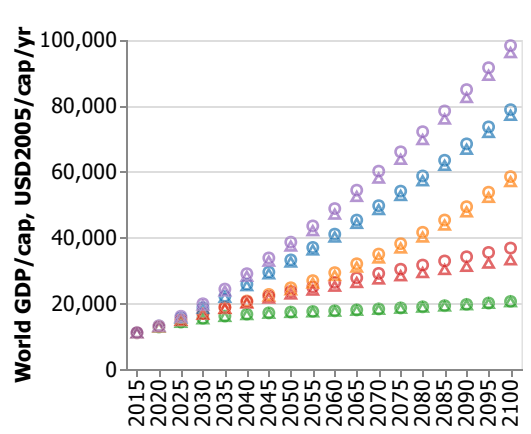

(a)

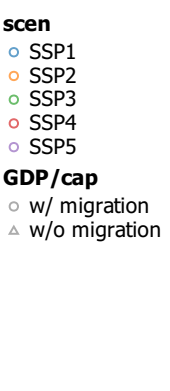

- SSP1

- SSP2

- SSP4

o $\mathrm{W} /$ migration

$\triangle$ w/o migration

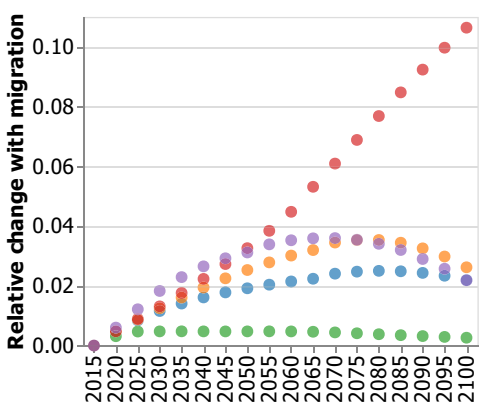

(b)

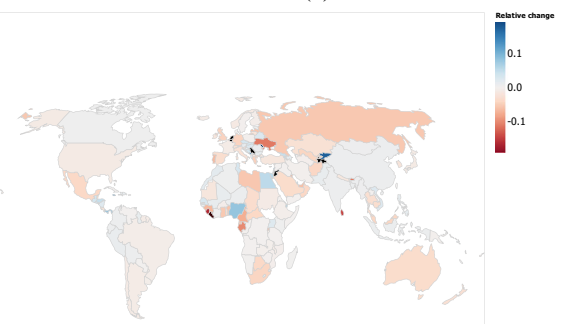

(c)

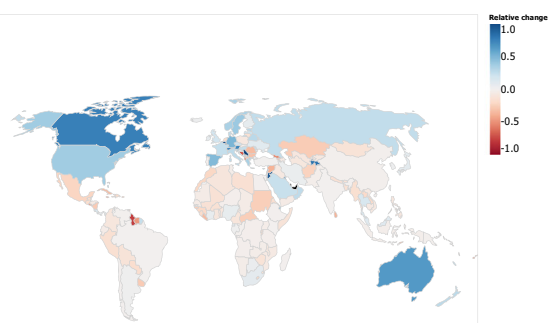

(d)

Fig. 1 Income with and without migration, for all SSP narratives. a Average world per capita GDP, in 2005 US dollars. b Average relative changes in per capita GDP with vs without migration (world). c Country-specific relative changes in per capita GDP with vs. without migration for SSP2 ("Middle of the Road") by 2100. d Country-specific relative changes in GDP with vs without migration for SSP2 by 2100

Migrants therefore have a strong incentive to move to richer countries, even in cases where the desired destination is far away. The remittances sent will likely amount to a significant share of their family income back home, thus contributing to an acceleration of income convergence across countries. In such a world, international migration is highly beneficial from a global income equalization perspective. On the other hand, in a strongly globalized world such as that depicted by SSP1, SSP2, and SSP5, income disparities across countries are relatively small by the end of the century. Migration will thus tend to take place, but mostly across countries within a close distance, with a relatively similar GDP per capita to that of the origin economy.

Figure $1 \mathrm{c}$ and $\mathrm{d}$ show relative changes in country-level per capita GDP and total GDP with migration compared to our zero-migration projections for the "Middle of the Road" SSP2 scenario by 2100 . We find that the extent and direction of the migration effect are highly country-specific. In general, destination countries (see, for example, Canada or United Arab Emirates) fare higher GDP levels with migration, as well as moderate changes in per capita GDP $( \pm 10 \%)$, with the sign reflecting net remittances received (see Eq. (1)). This means that some destination countries (e.g., Belgium, Jordan, or Lebanon) still receive net remittances, since their emigrants move to relatively rich countries and send back considerable income. For origin countries, the migration effect on GDP is less unidirectional. Some countries (e.g., Tajikistan, Serbia, or Nigeria) display higher income in the scenarios including migration, as remittances received from significantly richer countries are able to compensate for the loss of inhabitants contributing to the national economy. For the majority of origin countries (e.g., Guyana, Albania, Guinea-Bissau), this compensation does not take place as emigrants leave to 
countries that are not rich enough to provide high remittances to the origin economy. In some cases, origin countries are also net remittance-sending countries (e.g., Liberia, Sri Lanka, Kazakhstan), as their emigrants move to areas that may present less favorable income conditions than where their immigrants come from.

\subsection{GDP per capita projections for zero migration: continental outlook}

Figure 2 shows a decomposition of our GDP per capita projections at the continental level (see Section A of the Appendix for a definition of the world regions used). The effect of migration appears positive for Africa, Asia, and Oceania. In those regions, migration increases per capita income for all SSP narratives over the century by up to 5\%,6\%, and $21 \%$, respectively. Conversely, for Northern America and Europe, migration decreases per capita income by up to $1.4 \%$ and $5 \%$, respectively, by the end of the century. This effect diminishes over time in Northern America for SSP2, SSP3, and SSP5. Finally, for Latin America and the Caribbean, while for a start migration contributes to increasing income levels for all SSP narratives, this effect changes direction and migration ends up decreasing income levels by $1.5 \%$ for SSP1, SSP2, and SSP4, and by $4 \%$ in 2100 for SSP5. Overall, the effect of migration on income strongly depends on the particular SSP narrative considered. ${ }^{5}$

\subsection{Migration effects on between-countries inequality}

Our new GDP projections under the zero migration assumption allow us to address the role that migration plays as a determinant of income inequality between countries. To that aim, we use the global Gini coefficient, a commonly used measure of inequality, which considers the population-weighted distribution of income between countries. We project the global Gini coefficient based exclusively on between-countries inequality for all SSP narratives, both with and without migration, using our population and GDP projections. We show results in Fig. 3. Figure 3 a illustrates the global Gini coefficient with (circles) and without (triangles) migration, for all SSP narratives. While the effect of migration dynamics is visible, it stays moderate and does not modify the ranking of inequality levels among SSP narratives: SSP4 is still the scenario with the consistently highest inequality, while SSP5 and then SSP1 from around 2080 are the least unequal.

Figure $3 \mathrm{~b}$ shows relative changes in between-countries Gini coefficients with migration, compared to our zero-migration projections. We find that for all SSP narratives migration makes the world consistently more equal in terms of cross-country income differences by up to $6 \%$ by the end of the century. For SSP 2 and SSP4, the migration effect on between-countries inequality stays between 1.5 and $3 \%$ for the whole century, while for SSP1 and SSP3 it decreases to become zero by 2100 . Conversely for SSP5, the migration effect intensifies after 2070 and reaches $6 \%$ by 2100 . This substantial migration effect happens at a time when inequality starts increasing again and migration limits this increase. This differential effect of migration on inequality by the end of the century reflects the underlying hypotheses on migration levels and on worldwide convergence of income levels across the narratives. In an SSP5 world of global, strong, fossil-fueled economic growth, income levels are not

\footnotetext{
${ }_{5}^{5}$ The projections include a correction for four countries where net remittances become larger than income levels in the projection period. For these economies, we cap the ratio of net remittances to GDP once the projections imply negative income levels.
} 

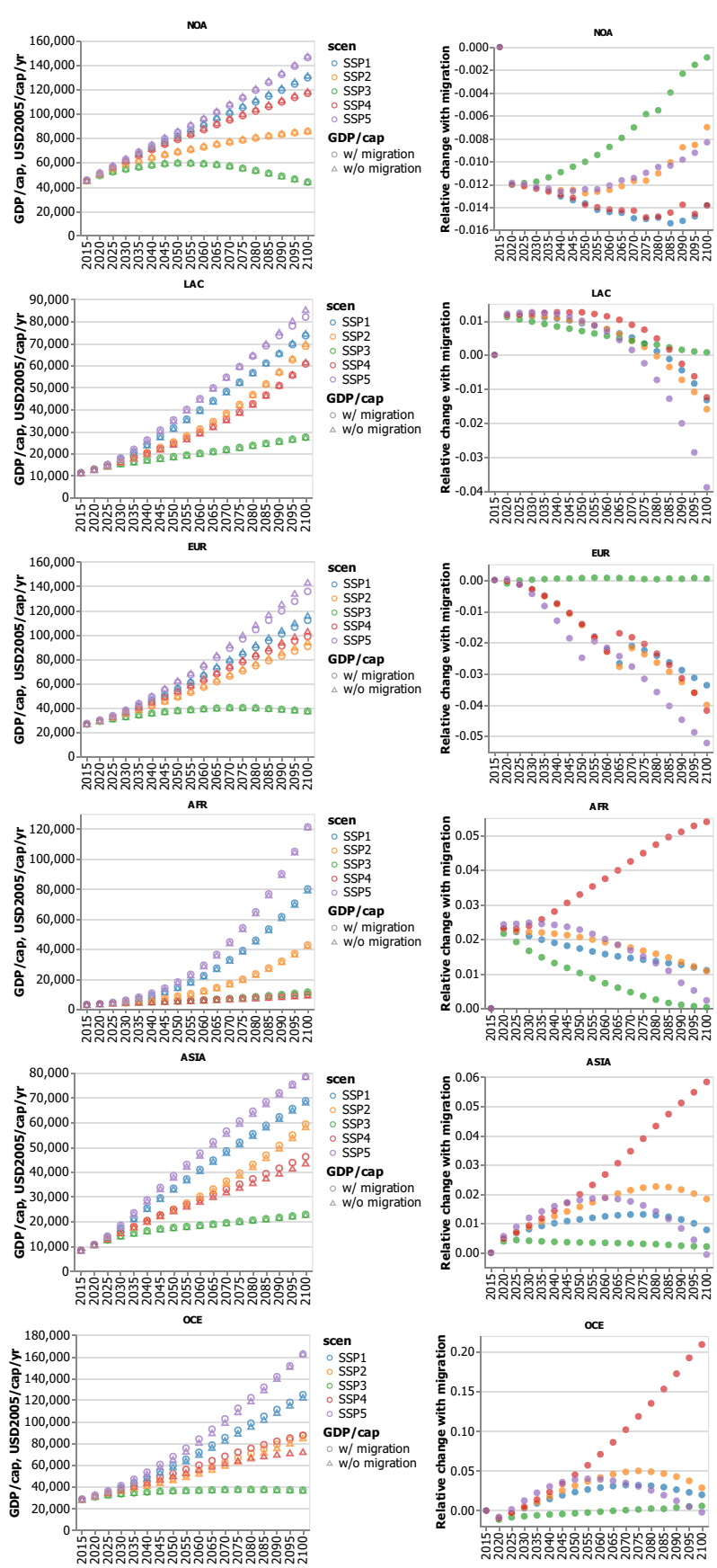

Fig. 2 Per capita GDP with and without migration, for all SSP narratives, averaged for major world regions. Left panel: Absolute values, in US dollars 2005 per capita per year. Right panel: Relative changes with vs. without migration. From top to bottom: World regions considered, following KC and Lutz (2017): Africa (AFR), Asia (ASIA), Europe (EUR), Latin America and the Caribbean (LAC), Northern America (NOA), Oceania (OCE) 


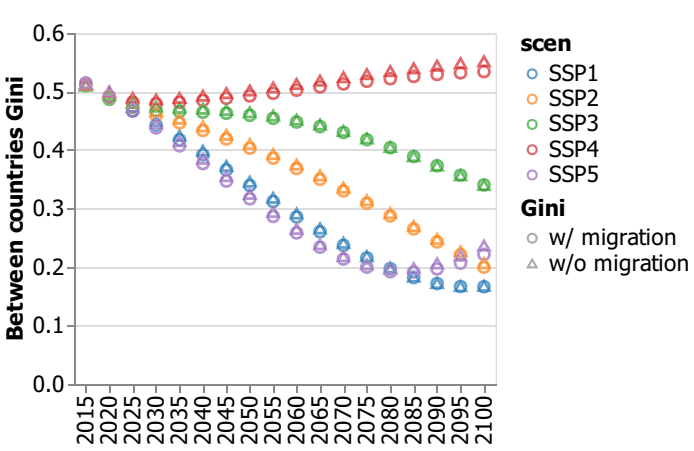

(a)

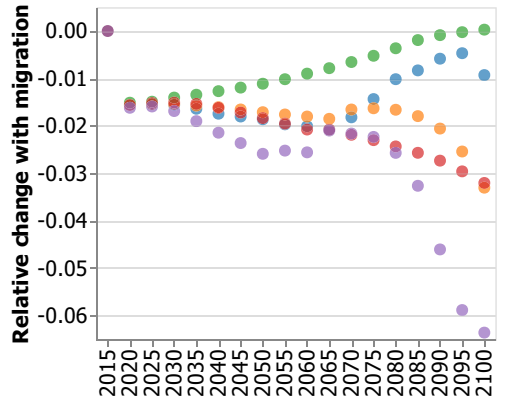

(b)

Fig. 3 World inequality as measured by between-countries Gini coefficients, for all SSP narratives. a Absolute Gini values with and without migration. b Relative changes in Gini coefficients comparing a scenario with migration to that without migration

substantially different across countries by 2100 , but migration levels are still high. Our results thus indicate that in general migration is expected to play a significant role in regulating inequality between countries over the coming decades, and much more so in scenarios where strong income differences persist.

\subsection{Migration effects on within-country inequality}

In order to obtain a more complete picture of the effect of migration on inequality, we also assess its role as a determinant of inequality within countries. To that aim, we project withincountry Gini coefficients under a zero-migration assumption using the specification proposed in Rao et al. (2019) (see Eq. (1) in their contribution) and compute changes in Gini stemming exclusively from migration-driven changes in education drivers (education levels and share of public spending), using their estimated coefficients (see Table 2 in Rao et al. (2019)). The model used in Rao et al. (2019) is not a structural model that would allow us to quantify the causal mechanisms underlying the interplay between education, income, and inequality dynamics. However, it provides elasticities emanating from a reduced-form model which has good in-sample fit and sensible out-of-sample projection paths for the inequality measure. For our exercise, we employ the SSP population projections without migration, available with detailed education levels. As in Rao et al. (2019), we also use data on educational attainment levels by age and sex in 2010 from the Wittgenstein Center, as well as data on per capita education spending from Statistics on Public Expenditures for Economic Development (SPEED) (International Food Policy Research Institute, 2015). For the latter, we use regional averages for countries with missing data. We show the projection results in Figs. 4 and 5.

Figure 4 depicts within-country Gini projections with and without migration for all SSP narratives, aggregated at the world region level (continent-specific median and interquartile range). The left column displays projections with migration by Rao et al. (2019). Unsurprisingly, SSP3 ("Regional Rivalry") and SSP4 ("Inequality") present the starkest inequalities and SSP1 ("Sustainability") and SSP5 ("Fossil-fueled Development") the lowest ones across continents. Latin America and the Caribbean and Africa present the widest range of withincountry inequality measures. The middle column displays our projections without migration. Looking at regional aggregates, migration appears to have little effect on within-country inequality, even for SSP5, the scenario that presents the largest migration flows. The right 
column displays relative changes in Gini coefficients in scenarios with migration compared to those without migration. We find that the effect of migration on within-country inequality varies significantly depending on the country and the SSP narrative considered. The strongest relative changes with migration happen for SSP5, which displays both the highest migration activity and the lowest Gini coefficient values. In general, within-country inequality tends to decrease with migration in Northern America and Europe and increase in Latin America and the Caribbean and Africa, and displays moderate changes in both directions in Asia and Oceania.

Figure 5 provides a more granular picture of the developments of income inequality within countries by illustrating relative changes in Gini coefficients by 2100 for SSP2 ("Middle of the Road") for projections with and without migration. Countries in shades of blue present a decrease in inequality with migration, while countries in shades of red present an increase. Migration appears to have minimal effect on most large economies (USA, China, India, Brazil, or Russia). Many destination countries (Canada, Australia, Western Europe, Saudi Arabia), on the other hand, see their inequalities reduced with migration. For some origin countries (Mali, Burkina Faso, Sudan, Afghanistan), migration appears to strongly increase inequality. Yet, for most origin countries, the effect of migration stays limited to under $5 \%$ in either direction.

\section{Discussion and conclusion}

This study provides the first quantification of international migration effects on income projections in the SSP framework. It highlights how migration effects strongly depend on context, both in terms of the country and the narratives considered for projection. We show that migration tends to make the world richer, on average, in all SSP scenarios. Furthermore, the choice of narrative - that is, the scenario of future development considered - significantly influences the nature of migration and remittance corridors. Depending on the narrative and location, the migration effect on income can be substantial, from -5 to $+21 \%$ at the continental level. Finally, we derive migration effects on inequality both between and within countries. We show that migration tends to make the world more equal in terms of between-countries income differentials under all SSP scenarios, has little effect on within-country income inequality in most major economies and origin countries, increases it in few origin countries, and reduces inequalities in most destination countries.

Limitations of this study derive from the characteristics of the model and data employed. We calibrate our gravity model to past and current data, but maintain the calibrated parameters constant over time and across the SSP narratives, since those dimensions are not quantified in the original SSP projections. Similarly, in our remittance model, we use current data for corridor-specific calibration, but keep the calibrated parameters constant over time and across the narratives. More importantly, we do not consider any heterogeneity of migrants or non-migrants within countries and abstract from the effects of potential differentials in productivity between migrants and local population. In fact, the socioeconomic characteristics of migrants tend to differ strongly across countries, over time, and even for the same origin country (de Haas et al. 2019). To the extent that brain drain may negatively affect productivity in the origin country and positively in the destination country, the between-countries inequalities we project may actually represent a lower bound to the actual effects of migration. Future research aimed at including such dimensions in our model should help shed light on particular aspects of international migration dynamics (the role of skill mismatch, for instance) in the projection exercises. 

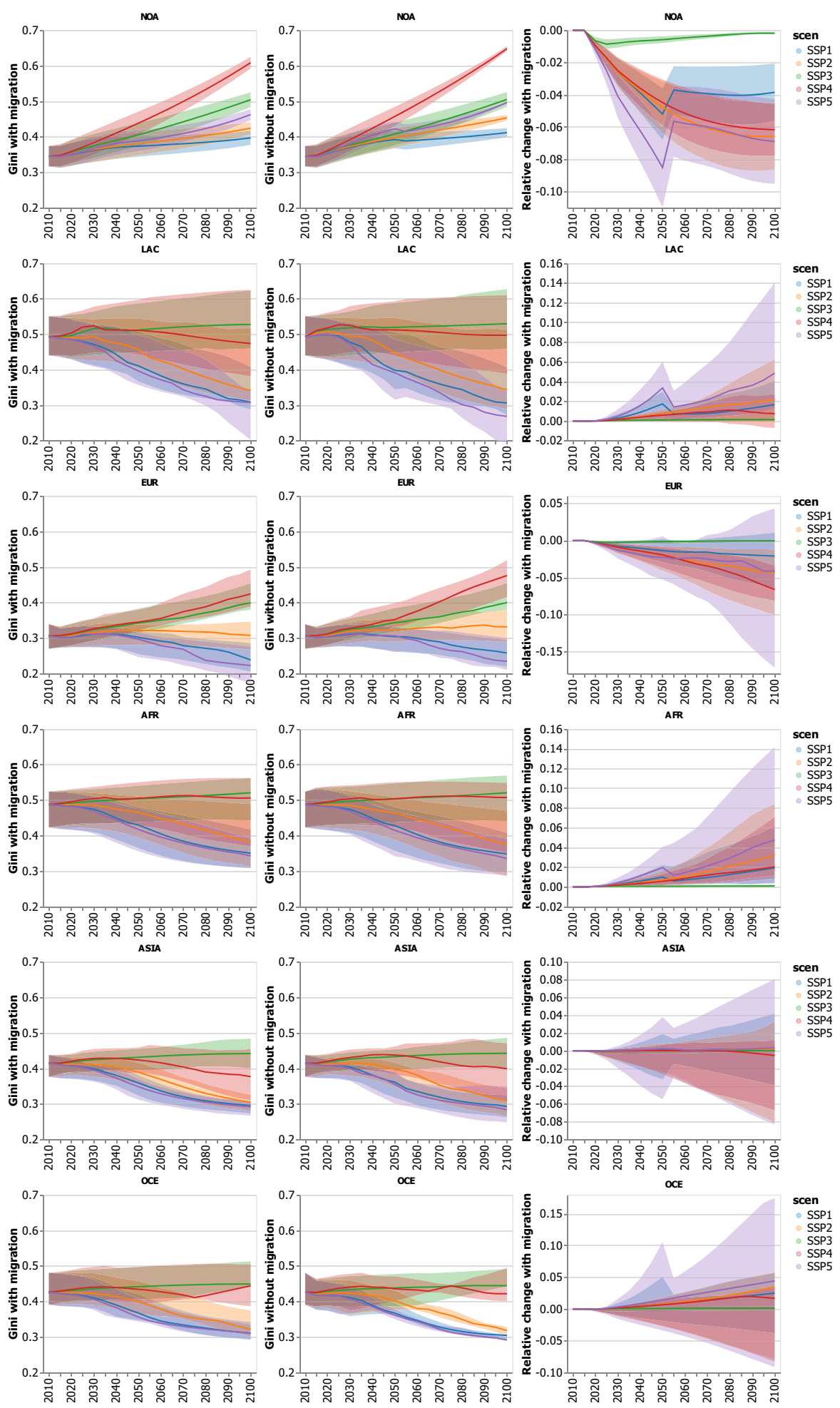
Fig. 4 Within-country inequalities as measured by within-country Gini coefficients with and without migration, for all SSP narratives. Median and interquartile range for major world regions. Left: Gini with migration. Center: Gini without migration. Right: Relative changes with vs without migration. From top to bottom: Northern America, Latin America and the Caribbean, Europe, Africa, Asia, Oceania

In line with the conceptual framework around the SSP projections, our aim for developing projections of various SSP components for zero migration is not to provide realistic forecasts of future developments, but rather to offer quantifications of migration consistent with the qualitative storyline for each SSP. Still, our analysis has direct policy implications. Despite its caveats, our study strongly suggests that any one-size-fits-all approach to projecting future international migration in a climate change context can be highly misleading, and conduce to misplaced policy answers. In such differentiated circumstances, a scenario framework is helpful, perhaps even necessary, to think about appropriate policy responses. Furthermore, our zero-migration projections fit perfectly in the interdisciplinary methodological framework of the SSP, and offer consistency with the original projections. This makes them particularly powerful to inform policymaking, in combination with other existing projections, for instance of migration policies. Future research in the further development of the SSPs or other similar frameworks could be enhanced by incorporating key aspects of the findings presented here. Migration effects on income and inequality can be directly included in the conceptualization of refined narratives, and our approach can be utilized to quantify such effects endogenously for next-generation socioeconomic projections. The incorporation of this study's findings in such projections would have particular impact on quantifications of adaptive capacity in relation to climate change scenarios, for example. Finally, including our projections as input scenarios in a climate-economy model with explicit migration dynamics, with the aim of

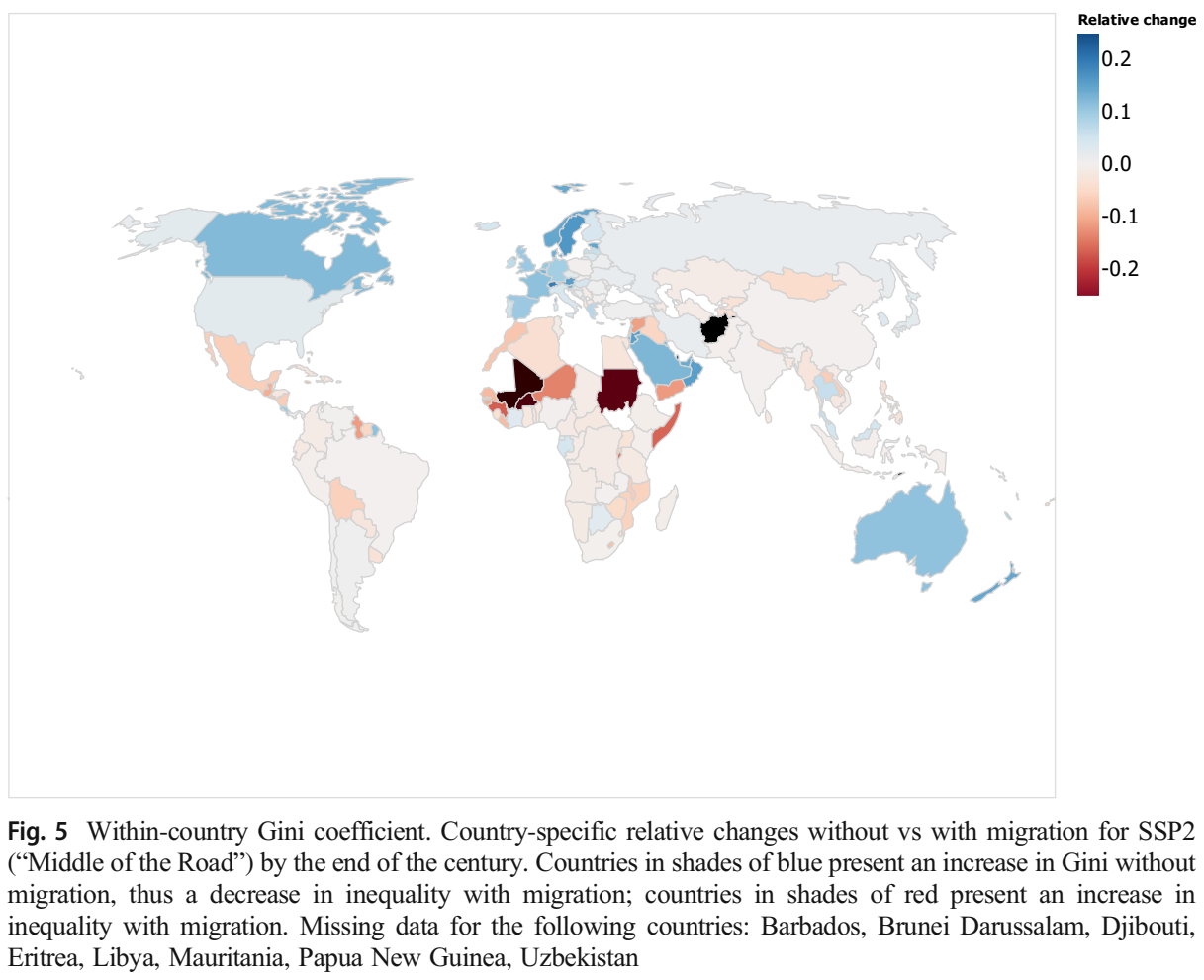


investigating interactions between migration and climate policies, appears as a natural avenue of further research.

Supplementary Information The online version contains supplementary material available at https://doi.org/ 10.1007/s10584-021-03133-w.

Acknowledgements The authors thank four anonymous reviewers for very helpful comments, as well as Samir $\mathrm{KC}$ and Guy Abel for support with the data and extremely valuable discussions on an earlier draft of the paper. Code availability Code is available upon request.

Author contribution All authors contributed to the study conception and design. Material preparation, data collection, and analysis were performed by HB and JCC. The first draft of the manuscript was written by HB and all authors commented on previous versions of the manuscript. All authors read, approved, and contributed to the writing of the final manuscript.

Funding Open access funding provided by International Institute for Applied Systems Analysis (IIASA). Part of this research was developed in the Young Scientists Summer Program at the International Institute for Applied Systems Analysis, Laxenburg (Austria) with financial support from the National Science Foundation through the National Academy of Sciences as the US National Member Organization. The authors gratefully acknowledge funding from IIASA and the National Member Organizations that support the institute.

Data availability Detailed projections at country level over 2015-2100 for all SSP narratives are available upon request to the authors for the following quantities:

- GDP for zero migration

- GDP per capita for zero migration

- Bilateral migrant flows

- Bilateral remittance flows

- Between-countries Gini coefficients for zero migration

- Within-country Gini coefficients for zero migration

\section{Declarations}

Conflict of interest The authors declare no competing interests.

Open Access This article is licensed under a Creative Commons Attribution 4.0 International License, which permits use, sharing, adaptation, distribution and reproduction in any medium or format, as long as you give appropriate credit to the original author(s) and the source, provide a link to the Creative Commons licence, and indicate if changes were made. The images or other third party material in this article are included in the article's Creative Commons licence, unless indicated otherwise in a credit line to the material. If material is not included in the article's Creative Commons licence and your intended use is not permitted by statutory regulation or exceeds the permitted use, you will need to obtain permission directly from the copyright holder. To view a copy of this licence, visit http://creativecommons.org/licenses/by/4.0/.

\section{References}

Abel GJ (2018a) Estimates of global bilateral migration flows by gender between 1960 and 2015. International Migration Review 52(3):809-852

Abel GJ (2018b) Non-zero trajectories for long-run net migration assumptions in global population projection models. Demographic Research 38(54):1635-1662

Abel GJ, Cohen JE (2019) Bilateral international migration flow estimates for 200 countries. Scientific Data 6(82) 
Adsera A, Pytlikova M (2015) The role of language in shaping international migration. The Economic Journal 125(586):F49-F81

Aldridge RW, Nellums LB, Bartlett S et al (2018) Global patterns of mortality in international migrants: a systematic review and meta-analysis. The Lancet 392:2553-2566. https://doi.org/10.1016/S0140-6736(18) 32781-8

Arezki R, Brückner M (2012) Rainfall, financial development, and remittances: evidence from Sub-Saharan Africa. Journal of International Economics 87:377-385

Azose JJ, Raftery AE (2015) Bayesian probabilistic projection of international migration. Demography 52:1627-1650

Azose JJ, Raftery AE (2019) Estimation of emigration, return migration, and transit migration between all pairs of countries. Proceedings of the National Academy of Sciences 116(1):116-122

Benveniste H, Oppenheimer M, Fleurbaey M (2020) Effect of border policy on exposure and vulnerability to climate change. Proceedings of the National Academy of Sciences 117(43):26692-26702. https://doi.org/10. 1073/pnas.2007597117

Black R, Adger N, Arnell N, Dercon S, Geddes A, Thomas D (2011) Foresight: migration and global environmental change

Black R, Bennett SRG, Thomas SM, Beddington JR (2011) Climate change: migration as adaptation. Nature 478:447-449

Borderon M, Sakdapolrak P, Muttarak R, Kebede E, Pagogna R, Sporer E (2019) Migration influenced by environmental change in Africa: a systematic review of empirical evidence. Demographic Research 41(18): 491-444

Calvin K, Bond-Lamberty B, Clarke L, Edmonds J, Eom J, Hartin C, Kim S, Kyle P, Link R, Moss R, McJeon H, Patel P, Smith S, Waldhoff S, Wise M (2017) The SSP4: a world of deepening inequality. Global Environmental Change 42:284-296

Castells-Quintana D, Lopez-Uribe MP, McDermott TKJ (2018) Adaptation to climate change: a review through a development economics lens. World Development 104:183-196

Cattaneo C, Peri G (2016) The migration response to increasing temperatures. Journal of Development Economics 122:127-146

Clemens MA, Pritchett L (2008) Income per natural: measuring development for people rather than places. Population and Development Review 34(3):395-434

Cohen JE, Roig M, Reuman DC, GoGwilt C (2008) International migration beyond gravity: a statistical model for use in population projections. Proceedings of the National Academy of Sciences 105(40):15269-15274

Crespo Cuaresma J (2017) Income projections for climate change research: a framework based on human capital dynamics. Global Environmental Change 42:226-236

De Haas H (2010) Migration and development: a theoretical perspective. International Migration Review 44: 227-264

De Haas H, Czaika M, Flahaux M-L et al (2019) International migration: trends, determinants, and policy effects. Population and Development Review 45:885-922

Dellink R, Chateau J, Lanzi E, Magne B (2017) Long-term economic growth projections in the shared socioeconomic pathways. Global Environmental Change 42:200-214

Desmet K, Kopp RE, Kulp SA, Nagy DK, Oppenheimer M, Rossi-Hansberg E, Strauss BH (2021) Evaluating the economic cost of coastal flooding. American Economic Journals: Macroeconomics 13(2):444-486

Fokkema T, Cela E, Ambrosetti E (2013) Giving from the heart or from the ego? Motives behind remittances of the second generation in Europe. International Migration Review 47(3):539-572. https://doi.org/10.1111/ imre. 12032

Fricko O, Havlik P, Rogelj J, Klimont Z, Gusti M, Johnson N, Kolp P, Strubegger M, Valin H, Amann M, Ermolieva T, Forsell N, Herrero M, Heyes C, Kindermann G, Krey V, McCollum DL, Obersteiner M, Pachauri S, Rao S, Schmid E, Schoepp W, Riahi K (2017) The marker quantification of the shared socioeconomic pathway 2: a middle-of-the-road scenario for the 21st century. Global Environmental Change 42:251-267

Fujimori S, Hasegawa T, Masui T, Takahashi K, Herran DS, Dai H, Hijioka Y, Kainuma M (2017) SSP3: AIM implementation of shared socioeconomic pathways. Global Environmental Change 42:268-283

Gemenne F, Blocher J (2017) How can migration serve adaptation to climate change? Challenges to fleshing out a policy ideal. The Geographical Journal 183:336-347

Hanson G, McIntosh C (2016) Is the Mediterranean the new Rio Grande? US and EU immigration pressures in the long run. Journal of Economic Perspectives 30(4):57-82

Hoffmann R, Dimitrova A, Muttarak R, Crespo Cuaresma J, Peisker J (2020) A meta-analysis of country-level studies on environmental change and migration. Nature Climate Change:1-9. https://doi.org/10.1038/ s41558-020-0898-6

International Food Policy Research Institute (2015) Statistics on Public Expenditures for Economic Development (SPEED). Country Level Data, Washington 
Jones B, O’Neill BC (2013) Historically grounded spatial population projections for the continental United States. Environmental Research Letters 8:044021

Jones B and B C O'Neill (2016) Spatially explicit global population scenarios consistent with the Shared Socioeconomic Pathways, Environmental Research Letters 11, 084003

Kanta KR, de Sherbinin A, Jones B, Bergmann J, Clement V, Ober K, Schewe J, Adamo S, McCusker B, Heuser S, Midgley A (2018) Groundswell: preparing for internal climate migration

KC S (2020) Updated demographic SSP4 and SSP5 scenarios complementing the SSP1-3 scenarios published in 2018. IIASA Working Paper, Laxenburg WP-20-016

KC S, Lutz W (2017) The human core of the shared socioeconomic pathways: population scenarios by age, sex and level of education for all countries to 2100. Global Environmental Change 42:181-192

Kriegler E, Bauer N, Popp A, Humpenöder F, Leimbach M, Strefler J, Baumstark L, Bodirsky BL, Hilaire J, Klein D, Mouratiadou I, Weindl I, Bertram C, Dietrich J-P, Luderer G, Pehl M, Pietzcker R, Piontek F, Lotze-Campen H, Biewald A, Bonsch M, Giannousakis A, Kreidenweis U, Maeller C, Rolinski S, Schultes A, Schwanitz J, Stevanovic M, Calvin K, Emmerling J, Fujimori S, Edenhofer O (2017) Fossil-fueled development (SSP5): an energy and resource intensive scenario for the 21st century. Global Environmental Change 42:297-315

Leimbach M, Kriegler E, Roming N, Schwanitz J (2017) Future growth patterns of world regions - a GDP scenario approach. Global Environmental Change 42:215-225

Liang S, Yang X, Qi J et al (2020) CO2 emissions embodied in international migration from 1995 to 2015. Environmental Science and Technology. https://doi.org/10.1021/acs.est.0c04600

Loi S, Hale JM (2019) Migrant health convergence and the role of material deprivation. Demographic Research 40:933-962. https://doi.org/10.4054/DemRes.2019.40.32

Lutz W, Amran G, Belanger A, Conte A, Gailey N, Ghio D, Grapsa E, Jensen K, Loichinger E, Marois G, Muttarak R, Potančoková M, Sabourin P, Stonawski M (2019) Demographic scenarios for the EU: migration, population and education. Publications Office of the European Union, Luxembourg

Lutz W, Goujon A, Samir KC, Stonawski M, Stilianakis N (2018) Demographic and human capital scenarios for the 21st century: 2018 assessment for 201 countries

Lutz W, KC S (2010) Dimensions of global population projections: what do we know about future population trends and structures? Philosophical Transactions of the Royal Society 365:2779-2791

Lutz W, Striessnig E (2015) Demographic aspects of climate change mitigation and adaptation. Population Studies 69:S69-S76

Mehta NK, Elo IT, Engelman M et al (2016) Life expectancy among U.S.-born and foreign-born older adults in the united states: estimates from linked social security and Medicare data. Demography 53:1109-1134. https://doi.org/10.1007/s13524-016-0488-4

O’Neill BC, Kriegler E, Ebi KL, Kemp-Benedict E, Riahi K, Rothman DS, van Ruijven BJ, van Vuuren DP, Birkmann J, Kok K, Levy M, Solecki W (2017) The roads ahead: narratives for shared socioeconomic pathways describing world futures in the 21 st century. Global Environmental Change 42:169-180

Ortega F, Peri G (2009) The causes and effects of international migrations: evidence from OECD countries 19802005. National Bureau of Economic Research 14833

Poprawe M (2015) On the relationship between corruption and migration: empirical evidence from a gravity model of migration. Public Choice 163(3):337-354

Qi W, Li G (2020) Residential carbon emission embedded in China's inter-provincial population migration. Energy Policy 136(111065)

Rao ND, Sauer P, Gidden M, Riahi K (2019) Income inequality projections for the shared socioeconomic pathways (SSPs). Futures 105:27-39

Riahi K, van Vuuren DP, Kriegler E, Edmonds J, O’Neill BC, Fujimori S, Bauer N, Calvin K, Dellink R, Fricko O, Lutz W, Popp A, Crespo Cuaresma J, Samir KC, Leimbach M, Jiang L, Kram T, Rao S, Emmerling J, Ebi K, Hasegawa T, Havlik P, Humpenöder F, Silva LAD, Smith S, Stehfest E, Bosetti V, Eom J, Gernaat D, Masui T, Rogelj J, Strefler J, Drouet L, Krey V, Luderer G, Harmsen M, Takahashi K, Baumstark L, Doelman JC, Kainuma M, Klimont Z, Marangoni G, Lotze-Campen H, Obersteiner M, Tabeau A, Tavoni M (2017) The Shared Socioeconomic Pathways and their energy, land use, and greenhouse gas emissions implications: an overview. Global Environmental Change 42:153-168

Singh GK, Miller BA (2004) Health, life expectancy, and mortality patterns among immigrant populations in the United States. Can J Public Health 95:I14-I21

van Vuuren DP, Stehfest E, Gernaat DE, Doelman JC, van den Berg M, Harmsen M, de Boer HS, Bouwman LF, Daioglou V, Edelenbosch OY, Girod B, Kram T, Lassaletta L, Lucas PL, van Meijl H, Maeller C, van Ruijven BJ, van der Sluis S, Tabeau A (2017) Energy, land-use and greenhouse gas emissions trajectories under a green growth paradigm. Global Environmental Change 42:237-250 
Wallace M, Khlat M, Guillot M (2019) Mortality advantage among migrants according to duration of stay in France, 2004-2014. BMC Public Health 19. https://doi.org/10.1186/s12889-019-6652-1

Publisher's note Springer Nature remains neutral with regard to jurisdictional claims in published maps and institutional affiliations.

\section{Affiliations}

\section{Hélène Benveniste ${ }^{1,2}$ • Jesús Crespo Cuaresma ${ }^{1,3,4,5}$ • Matthew Gidden ${ }^{1,6} \cdot$ Raya Muttarak $^{1,4}$}

1 International Institute for Applied Systems Analysis, Laxenburg, Austria

2 School of Public and International Affairs, Princeton University, Princeton, NJ, USA

3 Department of Economics, Vienna University of Economics and Business, Vienna, Austria

4 Wittgenstein Centre for Demography and Global Human Capital, IIASA, OeAW, University of Vienna, Vienna, Austria

5 Austrian Institute of Economic Research, Vienna, Austria

6 Climate Analytics, Berlin, Germany 